\title{
Opisthorchis felineus and Metorchis bilis Metacercariae in Cyprinid Fish Leuciscus idus in Nura-Sarysu River, Kazakhstan
}

\author{
Vladimir S. Kiyann 1,*, Aitbay K. Bulashev', Aleksey V. Katokhin ${ }^{2}$ \\ 'Department of Microbiology and Biotechnology, Saken Seifullin Kazakh Agrotechnical University, Astana, Kazakhstan; \\ 2Institute of Cytology and Genetics, Siberian Branch of the Russian Academy of Sciences, Novosibirsk, Russia
}

\begin{abstract}
Aim of the present study was to provide presence of opisthorchiid metacercariae in cyprinid fish Leuciscus idus in Nura-Sarysu river, Kazakhstan. Infection rate of the ides by the metacercariae was $42 \%$. The metacercariae, similar morphologically to those of the liver flukes, were found: elliptical in shape, $0.19-0.25 \times 0.15-0.22 \mathrm{~mm}$, oral and ventral suckers nearly equal size, and excretory bladder O-shape with black content, occupying posterior part of the body. The metacercariae were divided into 2 groups with differences in size and thickness of cyst wall. Adult flukes were recovered from the Syrian hamsters infected with the opisthorch metacercariae and identified with morphological characters to Opisthorchis felineus and Metorchis bilis. DNA sequences of ITS1, ITS2, and cox1 supported the taxonomic assignment.
\end{abstract}

Key words: Opisthorchis felineus, Metorchis bilis, Leuciscus idus, metacercariae

\section{INTRODUCTION}

Liver flukes of the family Opisthorchiidae (Looss, 1899) are considered as causative agents of serious disease worldwide, with the clinical pathology associated principally with chronic bile ducts infection with high risk of cholangiocarcinoma emergence [1-5]. All species within the family are obligate endoparasites with a complex 3-host life cycle including Bithyniidae snails and Cyprinidae fishes as the first and the second intermediate hosts and various piscivorous mammals and birds as definitive hosts [6,7].

Opisthorchiid flukes Opisthorchis felineus, O. viverrini, Clonorchis sinensis, and Metorchis bilis infect in the liver of mammals including humans $[5,8,2,9-12]$. Among these, $O$. felineus has been documented in humans and/or animals in 13 countries of the European Union [13]. This liver fluke also causes public health problem in the Russian Federation, Ukraine, Belarus and Kazakhstan [14].

Prevalence of opisthorchiasis reached a peak, 2,521 cases (17

\footnotetext{
- Received 6 November 2017, revised 18 May 2018, accepted 28 May 2018.

*Corresponding author (vskiyan@gmail.com)

(c) 2018, Korean Society for Parasitology and Tropical Medicine

This is an Open Access article distributed under the terms of the Creative Commons Attribution Non-Commercial License (http://creativecommons.org/licenses/by-nc/4.0) which permits unrestricted non-commercial use, distribution, and reproduction in any medium, provided the original work is properly cited.
}

cases per 100,000 population) in 2002 , and gradually decreased to 1,225 cases (7.4 cases per 100,000) in 2011 in Kazakhstan [15]. Infection cases with M. bilis have rarely been reported in Kazakhstan. In Russia O. felineus and M. bilis were the main agents of liver fluke infection of humans living in the Ob-Irtysh river basin. This river basin was the largest endemic area of opisthorchiasis $O$. felineus with 50-63\% of clinically diagnosed patients having mixed infection [11,16,17].

There are methods of diagnosing the disease but, have some drawbacks. Faecal examination for detection of parasite eggs and serological studies by ELISA are very laborious and fail to accurately distinguish the species of pathogen [18-22]. The development of highly sensitive and specific molecular genetics methods for the detection of trematodes of the family Opisthorchiidae and the precise identification of their species affiliation are an important urgent problem with a great epidemiological and medical significance [23-26]. Aim of this study was to identify the metacercariae of the family Opisthorchiidae in the Nura and Sarysu river basin Kazakhstan, using morphological and molecular genetic methods.

\section{MATERIALS AND METHOD}

\author{
Sample Collection \\ The 112 ides - Leuciscus idus (Linnaeus) (Cyprinidae) were
}


caught in Sholak Lake, located in Nura-Sarysu basin (Korgalzhyn district, Akmola region, Kazakhstan). The fishes were individually tested for the presence of the trematode metacercariae by the muscle compression method. Infected fish specimens were used to isolate metacercariae by the artificial digestion method [8].

\section{Experimental animals}

Ten Syrian golden hamsters (Mesocricetus auratus) at the age of 8-10 weeks were kept under good hygienic conditions at the vivarium of Saken Seifullin Kazakh Agrotechnical University (KATU), and their use and care were approved by the Animal Ethics Committee of Veterinary Medicine Faculty of KATU. All of the procedures were in compliance with the Code of Ethics of the World Medical Association (Declaration of Helsinki) for animal experiments (http://ec.europa.eu/environment/chemicals/lab_animals/legislation_en.htm). The hamsters were infected each orally with 50 metacercariae freshly isolated. The infection in the hamsters was verified by the coproovoscopy [27]. On the 40th day post infection (p.i.) hamsters were anesthetized with $\mathrm{CO}_{2}$ and euthanized, and adult trematodes were recovered from their livers for morphological and moleculargenetic studies.

\section{Molecular analyses}

For DNA extraction, 1/3 of the adult worm was homogenized separately in centrifuge tube and the method described previously [28]. DNA was dissolved in $\mathrm{ddH}_{2} \mathrm{O}$ and stored at $-70^{\circ} \mathrm{C}$. The polymerase chain reaction (PCR) was applied to identify opisthorchiid species using 3 primer pairs targeting nuclear ribosomal internal transcribed spacer 1 (ITS1: forward 5'-GTCGTAACAAGGTTTCCGTA-3' and reverse 5'-ACACGAGCCGAGTGATCC-3'), ITS2, (forward 5'-GAACATCGACATCTTGAACG-3', reverse 5'-GGAACGACCTGAACACCA-3') and mitochondrial DNA, cytochrome oxidase subunit 1 (cox1: forward 5'-GGGTTTGGAATGATTAGTC-3' and reverse 5'-CACAGAGGCAGAAAGAACT-3') [29-32]. As second step, amplicons of ITS1 and 2 others were sequenced and analysed for species identification. As controls, primer pairs of $O$. felineus and/or M. bilis were designed to amplify a part of the mitochondrial cox1 gene [33]. In addition, a primer pair was used as a control to detect of $O$. viverrini metacercariae from fish [34]. The primers were synthesized by a company (Integrated DNA technologies, Houston, Texas, USA).

Amplification of the targeted genes was carried out in a final reaction volume of $50 \mu \mathrm{l}$ containing $1 \times$ Phusion HF buffer, 2.5 $\mathrm{mM} \mathrm{MgCl} 2,1 \mathrm{U}$ Phusion DNA polymerase and $200 \mu \mathrm{M}$ dNTPs (New England BioLabs Inc., Ipswich, Massachusetts, USA), 25 pmol of each primer and 20 ng DNA extracted from a single liver fluke specimen as template.

PCR was performed as follows: denaturation at $95^{\circ} \mathrm{C}$ for 50 sec, primer annealing at $65^{\circ} \mathrm{C}$ for $50 \mathrm{sec}$, and extension at $68^{\circ} \mathrm{C}$ for $7 \mathrm{~min}$ with a final extension for $5 \mathrm{~min}$ at $72^{\circ} \mathrm{C}$. The resulting restriction fragments were separated by electrophoresis on an ethidium bromide containing 1.5\% agarose gel using $1 \mathrm{X}$ TAE buffer solution. A PCR-amplified target gene fragment was purified using E.Z.N.A. Gel Extraction kit (Omega Biotek, Norcross, Georgia, USA), following the manufacturer's protocols. The purified DNA was sequenced at the MD Anderson Cancer Centre, according to the Guidelines for DNA Submission. The resulting nucleotide sequences were visually checked by the Bio Capt program version 11.0. The nucleotide sequences of the studied species were compared with other sequences in the NCBI GeneBank database using BLAST. The phylogenetic analysis was carried out with MEGA 6 software.

The nucleotide sequences of the studied species were deposited in NCBI GenBank data base (O. felineus isolate 0827AKKz01: ITS1, MG952283, ITS2, MG952281; M. bilis isolate 0829-AKKz02: ITS1, MG952284, ITS2, MG952282).

\section{RESULTS}

\section{Morphology of the metacercariae}

The opisthorchiid metacercariae isolated from all they were identified by gross morphology: elliptical cyst shape, 0.19$0.25 \times 0.15-0.22 \mathrm{~mm}$ in size, equal size of oral and ventral suckers, and O-shaped excretory bladder filled with black excretory granules and occupying large part of posterior body $[8,11,35]$. Two species metacercariae were recognized morphologically very close, almost undistinguishable (Fig. 1). Their differences could be noted only in the size of cyst and thickness of cyst wall. Their variability of the features was overlapped, thus reduced differentiation capacity.

\section{Recovery of adult flukes}

Infection of the hamster was monitored daily after 30 days p.i. by coproovoscopy. The eggs of the opisthorchiid flukes were found in fecal pellets of the hamster on the 30-32 days p.i. Morphological features of the eggs were well compatible to opisthorchiid eggs. The eggs were light yellow or grayish, elon- 

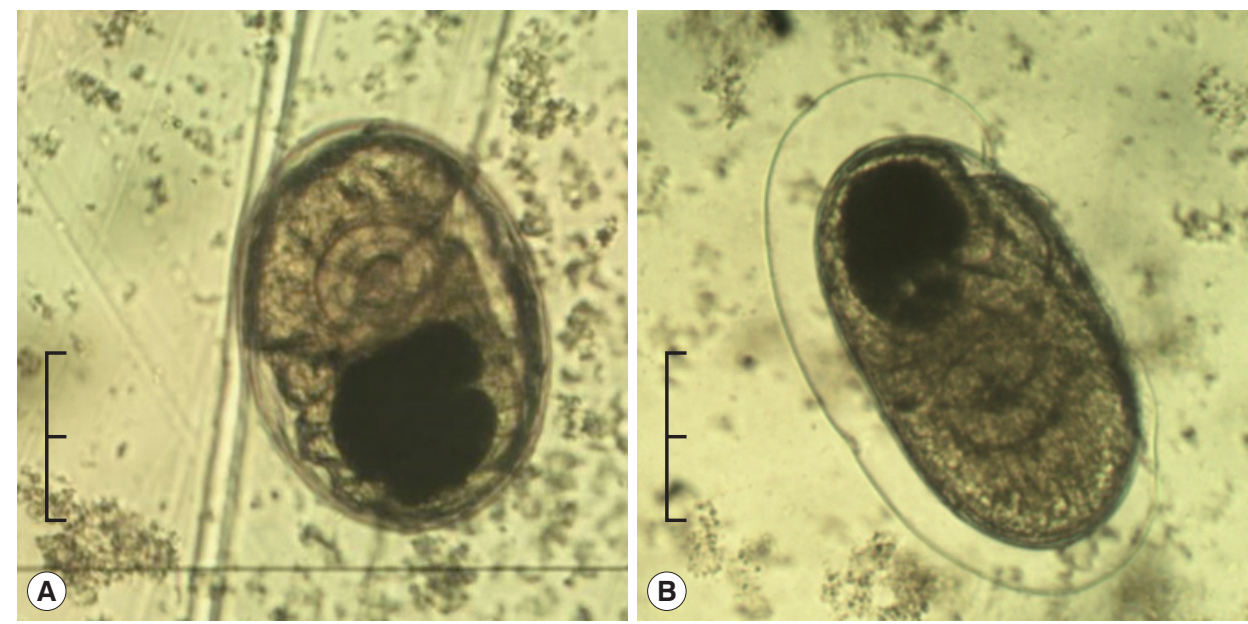

Fig. 1. Microphotographs of opisthorchiid metacercariae isolated from ides caught in sholak lake. (A) O. felineus. (B) M. bilis (Bar=0.1 mm).
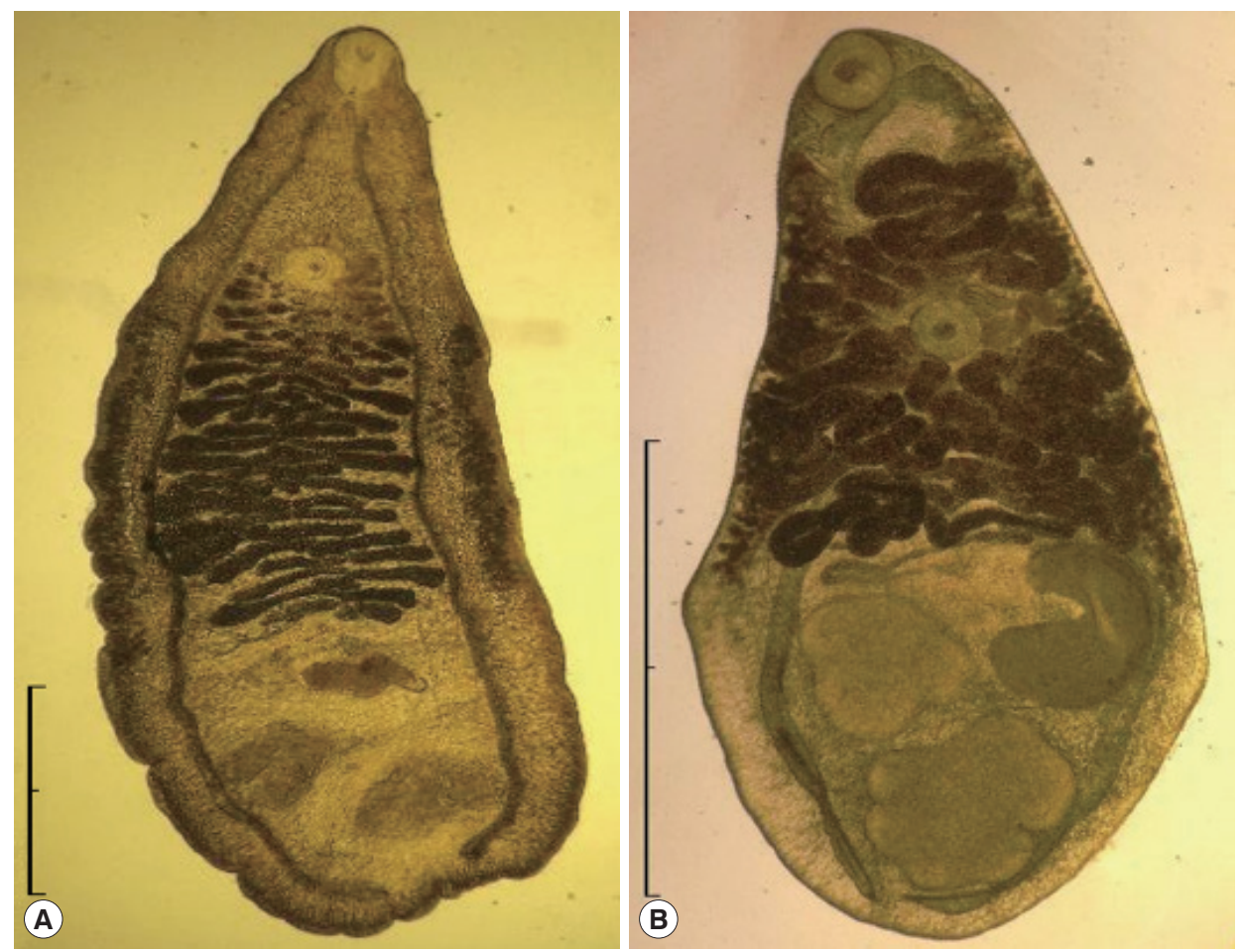

Fig. 2. Adult worms recovered from the infected hamsters. (A) O. felineus. (B) M. bilis (Bar=1 mm).

gated to oval, slightly asymmetric, having a lid on one end and a small bump on the other pole, measured 23.0-35.0 $\times 10.0$ $20.0 \mu \mathrm{m}$. Morphology of the eggs revealed high similarity in shape and size, thus it was hard to assign them to either opisthorchiid species.

At post-mortem examination on the 40th day p.i, adult liver flukes were recovered from the bile ducts and/or gall bladder of all infected hamsters, with a recovery rate $35 \pm 5.4 \%$
(17.5 \pm 2.7 flukes per hamster). The eggs collected after incubation of the recovered flukes were found as a mix of opisthorchiid eggs, very similar in shape and size. Based on the morphological features, the recovered adult flukes were identified to O. felineus or M. bilis (Fig. 2).

The $O$. felineus adult was lancet-shaped, narrow anteriorly to the end and rounded at the tail. Body size was 5-7 mm long and 1-1.5 mm wide [35]. The oral and ventral suckers were al- 
most equal in size. The oral sucker was round, diameter 0.26$0.28 \mathrm{~mm}$, subterminal, slightly flattened near the pharynx. The ventral sucker, round, diameter 0.24-0.25 mm, sited approximately anterior $1 / 5$ of the body. The pharynx, 0.14-0.16 $\mathrm{mm} \times 0.15-0.17 \mathrm{~mm}$, located just posterior to the oral sucker. The esophagus, 0.15-0.16 mm long, passed down and bifurcated into 2 intestinal caeca laid in the lateral fields and stretched to posterior end of the body. The ovary is smoothedged, weakly lobed and localized in front of the testis in middle of the body. The uterus wound up from the ovary to front edge of the ventral sucker. The genital pores opened at anterior side of the ventral sucker. The vitelline glands as 7-8 bunches, aligned long both marginal fields lateral to the intestinal caeca in middle of the body. The seminal receptacle was behind the ovary. The testes were 4-5 lobed and located diagonally one after another. The described morphological features of the fluke shown on Fig. 2A corresponded well to those of $O$. felineus.

The M. bilis adults had leaflike form, body size was $2-4 \mathrm{~mm}$ long and 1-1.5 mm wide (Fig. 2B). The oral and ventral suckers were almost equal in size. The oral sucker was round, diameter $0.22-0.28 \mathrm{~mm}$, subterminal. The ventral sucker, round, diameter $0.10-0.30 \mathrm{~mm}$, shifted forward from middle of the body. The pharynx was oval, diameter $0.12-0.13 \mathrm{~mm}$. The esophagus was very short, practically absent. The ovary was smooth-edged, rounded, localized in front of the testis and slightly lateral from central axis of the body. The uterus wound up from the ovary and overlaps the ventral sucker, reaching approximately the same level as the vitelline glands. The genital pores opened anterior to the ventral sucker. The vitelline glands distributed from front level of the anterior testis or ovary and reach half level between the 2 suckers. The seminal receptacle was behind the ovary, equal to or larger than the ovary. The anterior and posterior testes were smooth-edged and located obliquely in hind quarter of the body. The seminal vesicle was quite crooked [11,36]. Significant overlapping in morphology between M. bilis and other species in genus Metorchis was reported [36,37].

The trematodes recovered were identified by morphological features each to $O$. felineus and M. bilis.

\section{Molecular identification}

Five adult flukes each of $O$. felineus (1st group) and M. bilis (2nd group) were used for molecular phylogenetic analysis. The PCR products were separated on 1.5\% agarose gel by electrophoresis (Fig. 3). Primer pairs ITS1 and ITS2 allowed to amplify $664 \mathrm{bp}$ and $525 \mathrm{bp}$ fragments from $O$. felineus and $\mathrm{M}$. bilis, respectively. The PCR for cox 1 produced $260 \mathrm{bp}$ amplicon of $O$. felineus, but not of M. bilis (Fig. 3A, B).

The OF primer pair amplified a specific $200 \mathrm{bp}$ fragment from the O. felineus DNA but, did not from the M. bilis DNA. The $M B$ primer pair neither produce amplicon from $O$. felineus or from M. bilis (Fig. 3B).

The PCR-amplified DNA fragments of the 1st and 2nd groups were purified from agarose gel and sequenced. As a result, we successfully obtained ITS1, ITS2, cox1 sequences of $O$. felineus and M. bilis in Nura-Sarysu basin.

The BLAST analyses approved that the 1st group was highly similar to $O$. felineus and the 2nd group was highly similar to M. bilis [37].

These similarity wasillustrated with results of phylogenetic

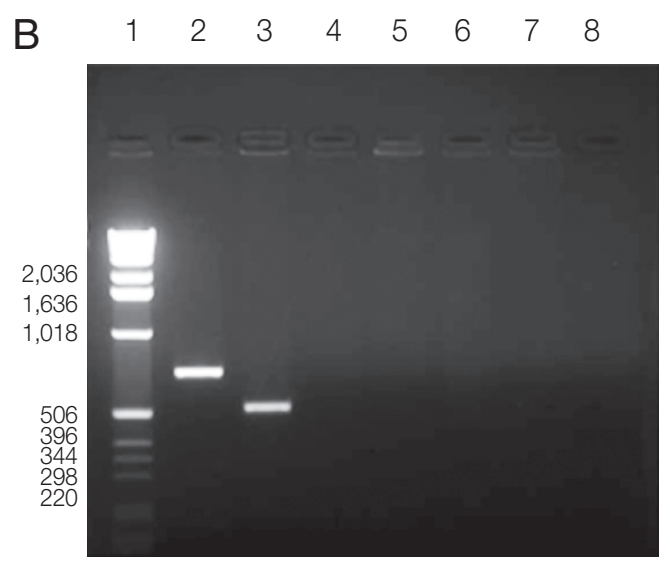

Fig. 3. Electrophoretic analysis of PCR products obtained with DNA of the adult trematodes. (A) Opisthorchis felineus. (B) Metorchis bilis: Lanes 2-7, primer pair of ITS1, ITS2, cox1, Of, Mb and Ov, respectively. Lane 1, DNA ladder (bp); lane 8, negative control. 


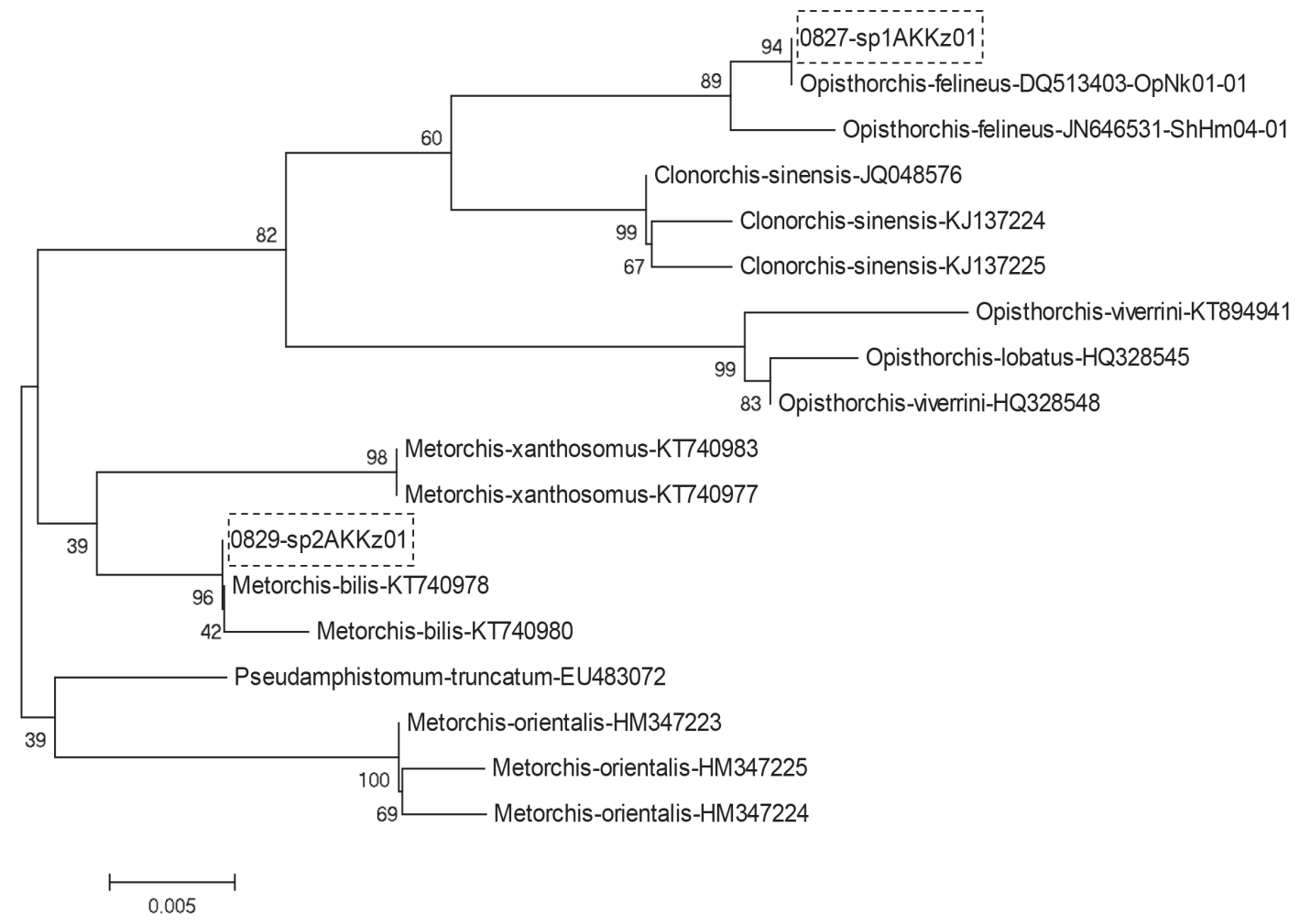

Fig. 4. Phylogenetic tree built out of opisthorchiids ITS1 sequences from GenBank and those produced in this work (indicated by boxes).

analysis using MEGA 6 software. The ITS1 phylogenetic tree constricted by ML algorithms certainly assigned the 1st group to $O$. felineus and the 2nd group to M. bilis (Fig. 4). The same result was obtained with ITS2 sequences of the 1st and 2nd groups (not shown).

\section{DISCUSSION}

In the Ob-Irtysh rivers basin - the world's largest endemic focus of $O$. felineus-associated opisthorchiasis, the majority of patients with clinical symptoms of opisthorchiasis were infected simultaneously with $O$. felineus and M. bilis $[11,24,25]$. Therefore, it is very important to have reliable methods for differentiation the metacercariae species in intermediate hosts for accurate epidemiologic prognosis and effective treatment and prevention measures development. The microscopic method of differentiating metacercariae is very laborious, time-consuming, and requires qualified specialists. The reliable methods that make possible to distinguish the larvae of trematodes from each other can be only based on molecular genetics approach [26].

Among the population living in the Nura-Sarysu basin, that is one of the 8 basins of Kazakhstan, opisthorchiasis is widespread, but mixed opisthorchiidiases have not been registered yet. If consider other intermediate hosts, there are only minor references to a mixed infection (O. felineus and M. bilis) in the study area. For example, necropsy investigations of village dogs in an endemic region revealed 37 of 51 (72\%) village dogs infected with either Opisthorchis felineus or Metorchis bilis. Two dogs were infected with single specimens of Metorchis bilis. However, the authors did not provide accurate (genetic) data on the presence of 2 species of parasite and emphasized that it was not possible to confirm whether the human opisthorchiidiasis was caused by O. felineus or M. bilis [15].

The present study provided the results of morphological and molecular identification of the trematodes of the family Opisthorchiidae circulating in the lakes of the Nura-Sarysu basin as metacercariae (encysted larvae) in ides. The choice of the fish species is explained by the data on previous studies reporting the Leuciscus idus living in the lakes of Nura-Sarysu basin to be the most infected with the metacercariae of the opisthorchiids [39]. Extensity of infection of the Leuciscus idus by Opisthorchiidae in the Sholak lakes of the Nura-Sarysu basin was $42 \%$.

The comparative description of morphology for O. felineus 
and M. bilis metacercariae was provided by S. Beer [8]: in encysted state they both have round or oval shape with thin transparent walls with size of $O$. felineus one being $0.17 \div 0.34 \times 0.21 \div 0.43$ $\mathrm{mm}$ versa $M$. bilis one being $0.13 \div 0.16 \times 0.19 \div 0.23$. Thus the 2 species encysted metacercariae are recognized as morphologically very close, almost undistinguishable [8]. Despite this, we managed to distinguish 2 different types, the differences of which were in size of cyst and thickness of cyst. But the differentiation of the 2 species flukes is more effective at their adult stage.

The hamsters experimentally infected with the larvae of opisthorchiids showed high susceptibility to infection. External and internal structure of the trematodes indicated that they belonged to 2 species: $O$. felineus and M. bilis. A molecular study was carried out to confirm the assignment.

In our study the previously described primers ITS1, ITS2 and cox 1 were used mainly for opisthorchiid DNA amplification [29-31]. The amplified DNA fragments of adult trematodes were then sequenced for species identification. It should be noted that we failed to amplify the DNA fragments of $M$. bilis not only by using cox1 primers, but also with the help of $M B$ primers which design was based on the sequence of $M$. $b i$ lis cox1 gene sequence [33]. The inability to amplify M. bilis DNA fragments using the primers mentioned requires further detailed study.

Thus, the ide living in the lakes of the Nura-Sarysu basin are additionally proved by molecular genetic technique to be invaded by metacercariae of 2 species of Opisthorchiidae trematodes infective for mammals including humans: $O$. felineus and M. bilis. These data indicate the high rate of mixed infection among the population of the Akmola region, which must be taken into account in the diagnosis and treatment of people from trematodes, as well as in the development of disease prevention measures.

\section{ACKNOWLEDGMENTS}

The authors are grateful to Dos D. Sarbassov (MD Anderson Cancer Center, Texas University, Houston, USA) who helped with specific primers and sequencing of PCR-amplified DNA fragments and Lyudmila A. Lider (S. Seifullin Kazakh AgroTechnical University, Astana, Kazakhstan) for their technical support in fish testing for the presence of metacercariae. This investigation was financially supported by the Ministry of Education and Science of the Republic of Kazakhstan within the frame-work of the projects No. 0115 RK00487 for 2015-2017, AP05131132 for 2018-2020. The molecular genetic work was partially supported by the project \#0324-2018-0016 of the FRC ICG SB RAS and the Russian Foundation for Basic Research (grant numbers 15-04-08893).

\section{CONFLICT OF INTEREST}

The authors declare that there are no competing interests related to the publication of this paper.

\section{REFERENCES}

1. IARC. Infection with liver flukes (Opisthorchis viverrini, Opisthorchis felineus and Clonorchis sinensis). IARC Monogr Eval Carcinog Risks Hum1994; 61: 121-175.

2. Sripa B, Bethony JM, Sithithaworn P, Kaewkes S, Mairiang E, Loukas A, Mulvenna J, Laha T, Hotez PJ, Brindley PJ. Opisthorchiasis and Opisthorchis - associated cholangiocarcinoma in Thailand and Laos. Acta Trop 2011; 120: 158-168.

3. Armignacco O, Ferri F, Gomez-Morales MA, Caterini L, Pozio E. Cryptic and asymptomatic Opisthorchis felineus infections. Am J Trop Med Hyg 2013; 88: 364-366.

4. Chai JY. Epidemiology of trematode infections. In Toledo R, Fried B eds, Digenetic Trematodes. New York, USA. Springer. 2014, pp 241-292.

5. Fedorova OS, Kovshirina YV, Kovshirina AE, Fedotova MM, Deev IA, Petrovskiy FI, Filimonov AV, Dmitrieva AI, Kudyakov LA, Saltykova IV, Odermatt P, Ogorodova LM. Opisthorchis felineus infection and cholangiocarcinoma in the Russian Federation: a review of medical statistics. Parasitol Int 2016; 66: 365-371.

6. King S, Scholz T. Trematodes of the family Opisthorchiidae: a minireview. Korean J Parasitol 2001; 39: 209-221.

7. Kaewkes S. Taxonomy and biology of liver flukes. Acta Trop 2003; 88: 177-186.

8. Beer SA. Biology of opisthorchosis invasion agent. 1st ed. Moscow. 2005, pp 115-164.

9. Kaewpitoon N, Kootanavanichpong N, Kompor P, Chavenkun W, Kujapun J, Norkaew J, Ponphimai S, Matrakool L, Tongtawee T, Panpimanmas S, Rujirakul R, Padchasuwan N, Pholsripradit P, Eksanti T, Phatisena T, Loyd RA, Kaewpitoon SJ. Review and Current Status of Opisthorchis viverrini Infection at the Community Level in Thailand. Asian Pac J Cancer Prev 2015; 16: 68256830.

10. Tang ZL, Huang Y, Yu XB. Current status and perspectives of Clonorchis sinensis and clonorchiasis: epidemiology, pathogenesis, omics, prevention and control. Infect Dis Poverty 2016; 5: 71.

11. Mordvinov VA, Yurlova NI, Ogorodova LM, Katokhin AV. Opisthorchis felineus and Metorchis bilis are the main agents of liver fluke infection of humans in Russia. Parasitol Int 2012; 61: 2531. 
12. Schuster RK. Opisthorchiidosis--a review. Infec Disord Drug Targets 2010; 10: 402-415.

13. Pozio E, Armignacco O, Ferri F, Gomez Morales MA. Opisthorchis felineus, an emerging infection in Italy and its implication for the European Union. Acta Trop 2013; 126: 54-62.

14. WHO. Control of food borne trematode infections. Report of a WHO study group. World Health Organ Tech Rep Ser 1995; 849: 1-157.

15. Sultanov A, Abdybekova A, Abdibaeva A, Shapiyeva Z, Yeshmuratov T, Torgerson PR. Epidemiology of fish-borne trematodiasis in Kazakhstan. Acta Trop 2014; 138: 60-66.

16. Kuznetsova VG, Naumov VA, Belov GF. Methorchiasis in the residents of Novosibirsk area, Russia. Cytobios 2000; 102: 33-34.

17. Fedorov KP, Naumov VA, Kuznetsova VG, Belov GF. Some real problems of human opisthorchiasis. Med Parazitol (Mosk) 2002; 3: 7-9 (in Russian).

18. Teimoori S, Arimatsu Y, Laha T, Kaewkes S, Sereerak P, Sripa M, Tangkawattana S, Brindley PJ, Sripa B. Chicken IgY-based coproantigen capture ELISA for diagnosis of human opisthorchiasis. Parasitol Int 2017; 66: 443-447.

19. Bulashev AK, Borovikov SN, Serikova SS, Suranshiev ZA, Kiyan VS, Eskendirova SZ. Development of an ELISA using anti-idiotypic antibody for diagnosis of opisthorchiasis. Folia Parasitol (Praha) 2016; 63: 025.

20. Waikagul J, Dekumyoy P, Chaichana K, Thairungroje Anantapruti M, Komalamisra C, Kitikoon V. Serodiagnosis of human opisthorchiasis using cocktail and electroeluted Bithynia snail antigens. Parasitol Int 2002; 51: 237-247.

21. Starkova TV, Poletaeva OG, Kovrova EA, Krasovskaia NN, Tkachenko TN, Masiago AV, Ofitserov VI, Tereshchenko Alu. The efficiency of the enzyme immunoassay test system opisthorchiasis-CIC-EIA-best to detect circulating immune complexes containing opisthorchis antigens in the serum of patients with opisthorchiasis. Med Parazitol (Mosk) 2011; 3: 44-45 (in Russian).

22. Nöckler K, Dell K, Schuster R, Voigt WP. Indirect ELISA for the detection of antibodies against Opisthorchis felineus (Rivolta, 1884 ) and Metorchis bilis (Braun, 1790) in foxes. Vet Parasitol 2003; 110: 207-215.

23. Müller B, Schmidt J, Mehlhorn H. PCR diagnosis of infections with different species of Opisthorchiidae using a rapid clean-up procedure for stool samples and specific primers. Parasitol Res 2007; 100: 905-909.

24. Aung WPP, Htoon TT, Tin HH, Thinn KK, Sanpool O, Jongthawin J, Sadaow L, Phosuk I, Rodpai R, Intapan PM, Maleewong W. First report and molecular identification of Opisthorchis viverrini infection in human communities from Lower Myanmar. PLoS One 2017; 12: e0177130.

25. Sahu R, Biswal DK, Roy B, Tandon V. Molecular characterization of Opisthorchis noverca (Digenea: Opisthorchiidae) based on nuclear ribosomal ITS2 and mitochondrial CO1 genes. J Helminthol 2016; 90: 607-614.

26. Brusentsov II, Katokhin AV, Sakharovskaia ZV, Sazonov AE, Ogorodova LM, Fedorova OS, Kolchanov NA, Mordvinov VA.
DNA diagnosis of mixed invasions of Opisthorchis felineus and Metorchis bilis by polymerase chain reaction. Med Parazitol (Mosk) 2010; 2: 10-13 (in Russian).

27. Pavliukobv IA, Berezantsev IuA. The ethyl acetate method of studying feces for Opisthorchis eggs. Med Parazitol (Mosk) 1991; 3: 57 (in Russian).

28. Boom R, Sol CJ, Salimans MM, Jansen CL, Wertheim-van Dillen PM, van der Noordaa J. Rapid and simple method for purification of nucleic acids. J Clin microbial 1990; 28: 495-503.

29. Müller B, Schmidt J, Mehlhorn H. Sensitive and species-specific detection of Clonorchis sinensis by PCR in infected snails and fishes. Parasitol Res 2007; 100: 911-914.

30. Kang S, Sultana T, Loktev VB, Wongratanacheewin S, Sohn WM, Eom KS, Park JK. Molecular identification and phylogenetic analysis of nuclear rDNA sequences among three opisthorchid liver fluke species (Opisthorchiidae: Trematoda). Parasitol Int 2008; 57: 191-197.

31. Katokhin AV, Shekhovtsov SV, Konkow S, Yurlova NI, Serbina EA, Vodianitskai SN, Fedorov KP, Loktev VB, Muratov IV, Ohyama F, Makhnev TV, Pel'tek SE, Mordvinov VA. Assessment of the genetic distinctions of Opisthorchis felineus from O. viverrini and Clonorchis sinensis by ITS2 and CO1 sequences. Dokl Biochem Biophys 2008; 421: 214-217.

32. Brusentsov II, Katokhin AV, Brusentsova IV, Shekhovtsov SV, Borovikov SN, Goncharenko GG, Lider LA, Romashov BV, Rusinek OT, Shibitov SK, Suleymanov MM, Yevtushenko AV, Mordvinov VA. Low genetic diversity in wide-spread Eurasian liver fluke Opisthorchis felineus suggests special demographic history of this trematode species. PLoS One 2013; 8: 62453.

33. Pauly A, Schuster R, Steuber S. Molecular characterization and differentiation of opisthorchiid trematodes of the species Opisthorchis felineus (Rivolta, 1884) and Metorchis bilis (Braun, 1790) using polymerase chain reaction. Parasitol Res 2003; 90: 409414.

34. Parvathi A, Umesha KR, Kumar S, Sithithaworn P, Karunasagar I, Karunasagar I. Development and evaluation of a polymerase chain reaction (PCR) assay for the detection of Opisthorchis viverrini in fish. Acta Trop 2008; 107: 13-16.

35. Romashov BV, Romashov VA, Semenov VA, Filimonova LV. Opisthorchiasis in the Upper Don Basin (Voronezh Oblast): The liver fluke fauna, ecological and biological patterns of circulation and foci of Opisthorchiases. 1st ed. Voronezh, Russia. Voronezh State University. 2005, pp 201 (in Russian).

36. Filimonova LV. A taxonomic review of two subfamilies (Metorchiinae Luhe, 1909 and Pseudamphistominae Yamaguti, 1958) of the family Opisthorchiidae Faust 1929 of the Russian fauna. Top Probl Parasitol Sci 1998; 42: 244-253.

37. Sitko J, Bizos J, Sherrard-Smith E, Stanton DW, Komorová P, Heneberg P. Integrative taxonomy of European parasitic flatworms of the genus Metorchis Looss, 1899 (Trematoda: Opisthorchiidae). Parasitol Int 2016; 65: 258-267.

38. Brusentsov II, Katokhin AV, Sakharovskaia ZV, Sazonov AE, Ogorodova LM, Fedorova OS, Kolchanov NA, Mordvinov VA. 
DNA diagnosis of mixed invasions of Opisthorchis felineus and Metorchis bilis by polymerase chain reaction. Med Parazitol (Mosk) 2010; 2: 10-13 (in Russian).

39. Kiyan VS, Bulashev AK, Tokpan SS, Baibolin ZhK, Alyoshina A.
The study of opisthorchiasis infection in the Akmola region. Bulletin of ENU LN Gumilev. Biology series 2015; 6: 134-142 (in Russian). 